Arts

et Savoirs

\section{Arts et Savoirs}

$12 \mid 2019$

Révolution et évolution

\title{
L'évolution des espèces sociales dans La Comédie humaine de Balzac
}

The Evolution of Social Species in Balzac's Comédie humaine

\section{Sandra Collet}

\section{(2) OpenEdition}

\section{Journals}

Édition électronique

URL : http://journals.openedition.org/aes/2317

DOI : $10.4000 /$ aes. 2317

ISSN : 2258-093X

Éditeur

Laboratoire LISAA

\section{Référence électronique}

Sandra Collet, "L'évolution des espèces sociales dans La Comédie humaine de Balzac », Arts et Savoirs [En ligne], 12 | 2019, mis en ligne le 27 février 2020, consulté le 02 mars 2020. URL : http:// journals.openedition.org/aes/2317 ; DOI : 10.4000/aes.2317

Ce document a été généré automatiquement le 2 mars 2020

Centre de recherche LISAA (Littératures SAvoirs et Arts) 


\title{
L'évolution des espèces sociales dans La Comédie humaine de Balzac
}

\author{
The Evolution of Social Species in Balzac's Comédie humaine
}

\author{
Sandra Collet
}

1 L'intérêt de Balzac pour l'histoire comme pour les sciences de la nature et du vivant médecine, physique, sciences naturelles - ne s'est jamais démenti : l'hybridation des savoirs historiques et biologiques est placée au cœur de la démarche du romancier, qui régulièrement présente l'Histoire et les sciences naturelles comme ses deux modèles épistémologiques. Balzac n'entend pas seulement faire œuvre de poète, mais aussi de savant; histoire et science naturelles doivent lui fournir les outils non seulement pour décrire de manière raisonnée et organisée les particularités de la société française de la première moitié $\mathrm{du} \mathrm{XIX}^{\mathrm{e}}$ siècle, mais aussi pour l'analyser et la comprendre, en conférant à son œuvre romanesque une autorité quasi-scientifique. Cette revendication est affichée dans L'Avant-Propos de la Comédie humaine, rédigé en 1842 pour réunir tous les romans écrits et à venir en un ensemble cohérent. Balzac y présente son programme : la peinture de la société et des mœurs d'une époque dont le romancier a vivement senti le caractère unique et original - bouleversée qu'elle fut par «trois systèmes différents qui ont en cinquante ans donné une physionomie spéciale aux meubles, aux habitations" (Une Fille d'Ève - II, 265) ${ }^{1}$. L'aristocratie, en particulier, brutalement confrontée à un complet renversement des valeurs et des mœurs, se voit, dans ce siècle, condamnée à se transformer, s'adapter - ou disparaitre : situation qui devient un thème récurrent dans les récits balzaciens.

Près de vingt ans avant l'émergence du débat darwinien et de ses prolongements sociologiques par Spencer, Balzac dresse le tableau d'une société humaine livrée à la compétition permanente, à la lutte pour la survie : l'adaptation au milieu, la sélection du meilleur sont des enjeux vitaux. On se propose d'analyser ici cette peinture sociale balzacienne, en examinant l'influence conjuguée des sciences naturelles et de l'histoire, en particulier sur la question de l'évolution des individus et des classes sociales, dans une société en pleine mutation. La classe aristocratique étant celle pour qui la question se pose de la manière la plus décisive, on limitera l'étude à cette seule catégorie, pour 
voir comment son devenir est envisagé et mis en question dans les romans balzaciens. Nous verrons comment Balzac a dépeint, et surtout cherché à analyser et comprendre, ces mécanismes sociaux, en s'appuyant sur les concepts et les outils de l'histoire et des savoirs biologiques.

Commençons par rappeler précisément l'état des connaissances scientifiques de Balzac, et par recenser les savoirs qu'il exploite.

4 On sait que l'auteur de La Comédie humaine s'est voulu historien plus encore que romancier. Ambitieux, il entend révolutionner - ou compléter - les méthodes historiques par une démarche novatrice : attaché aux petits faits vrais, révélateurs de l'atmosphère d'une époque, il prétend « écrire l'histoire oubliée par tant d'historiens, celle des mœurs. » (Avant-Propos de la Comédie humaine, I, 11). Cette démarche originale, par le champ auquel il entend se consacrer, se combine à une méthodologie moderne : informé des transformations qui agitent la discipline au même moment, Balzac se donne pour mission d'analyser et expliquer les phénomènes, de dégager la logique de causalité à l'œuvre dans les événements : « étudier les raisons ou la raison de ces effets sociaux, surprendre le sens caché dans cet immense assemblage de figures, de passions et d'événements" (Ibid.) De là vient la structuration tripartite de La Comédie humaine : les Études de mours décrivent les faits, tandis que les Études philosophiques exposent les causes et que les Études analytiques s'efforcent de remonter aux principes ${ }^{2}$; cette organisation doit tout à la science historique telle qu'elle s'élabore au XIX ${ }^{e}$ siècle.

5 Toutefois, quand il s'agit d'accomplir concrètement cette mission qu'il s'est assigné, la méthode historique trouve ses limites aux yeux du romancier: "vous ne pouvez raconter chronologiquement que l'histoire du temps passé, système inapplicable à un présent qui marche » note l'auteur dans la Préface d'Une Fille d'Ève (II, 265). Le modèle historique fait défaut dès lors que l'on entend décrire le présent, analyser l'état contemporain d'une société en perpétuel mouvement. Ces failles du modèle historique expliquent peut-être le travail d'hybridation opéré par le romancier, qui se tourne vers un autre modèle scientifique. Les sciences naturelles, et la toute jeune biologie, offrent des outils précieux pour penser les changements qu'enregistre la société française postrévolutionnaire, et élaborer une description raisonnée et analytique du temps présent.

Grand admirateur de Cuvier et de Geoffroy Saint Hilaire, mais aussi de Linné et de Buffon, c'est sous l'égide de ces augustes modèles que se place Balzac. La Comédie humaine sera le prolongement romanesque des nomenclatures du vivant réalisées par les savants du Museum d'histoire naturelle, dont les méthodes sont appliquées par Balzac à l'étude de la société française : «Si Buffon a fait un magnifique ouvrage en essayant de représenter dans un livre l'ensemble de la zoologie, n'y avait-il pas une œuvre de ce genre à faire pour la Société ?» $(\mathrm{I}, 8)$. Reprenant le postulat d'une analogie entre l'Humanité et l'Animalité, le romancier élabore le concept d'" Espèces sociales ", qui structureraient la société comme les espèces animales organisent le monde vivant:

La Société ne fait-elle pas de l'homme, suivant les différents milieux où son action se déploie, autant d'hommes différents qu'il y a de variétés en zoologie? Les différences entre un soldat, un ouvrier, un administrateur, un avocat, un oisif, un savant, un homme d'état, un commerçant, un marin, un poète, un pauvre, un prêtre, sont, quoique plus difficiles à saisir, aussi considérables que celles qui distinguent le loup, le lion l'âne, le corbeau, le requin, le veau marin, la brebis, etc. Il a donc existé, il existera de tout temps des Espèces Sociales comme il y a des Espèces Zoologiques. (I, 8) 
7 Toutefois, le recours à ce modèle épistémologique, comme celui de l'Histoire, n'est pas sans poser des difficultés au romancier. En particulier, les soubresauts de l'histoire récente imposent à Balzac de s'intéresser à un phénomène nouveau : la perméabilité des classes sociales, qui permet que «l'épicier devien[ne] certainement pair de France, et [que] le noble descend[e] parfois au dernier rang ${ }^{3}$ (I, 9). Et voilà Balzac confronté, presque comme malgré lui, aux débats brûlants qui animent les milieux naturalistes autour de la question l'évolution des espèces, depuis la célèbre controverse qui opposa au printemps 1830, devant l'Académie des sciences, Cuvier, tenant du fixisme, et Geoffroy Saint Hilaire, partisan de l'évolutionnisme.

Quel est l'état des connaissances du romancier sur la question? Si l'évolution des espèces sociales est un moteur romanesque récurrent dans les récits de La Comédie humaine, ce n'est cependant pas chez Lamarck que Balzac trouve les fondements théoriques susceptibles d'étayer sa réflexion: le nom de ce savant, mort dans l'indifférence quasi générale en décembre 1829, à une date où Balzac témoigne encore peu d'intérêt pour les sciences du vivant, n'apparaît pas une seule fois sous la plume du romancier. C'est bien plutôt aux savants du Museum, Cuvier et Geoffroy Saint Hilaire, qu'il doit ses connaissances : ces « deux génies égaux » (Illusions perdues, V, 317) sont les principales références du romancier, nonobstant leurs positions radicalement opposées dans le débat sur l'évolution.

9 Madeleine Fargeaud ${ }^{4}$ note que Geoffroy Saint Hilaire semble, au premier abord, avoir exercé l'influence la plus forte sur le romancier. Leur rencontre, au printemps 1835, déclencha l'admiration enthousiaste de Balzac, dont rend compte la dédicace du Père Goriot quelques semaines plus tard, adressée " au grand et illustre Geoffroy Saint Hilaire, comme un témoignage d'admiration de ses travaux et de son génie ». (Le Père Goriot - III, 49) $)^{5}$ Ailleurs, le savant est qualifié d' " homme de génie au premier chef » et $d^{\prime}$ " inventeur $»^{6}$. Ses travaux de classification du vivant inspirent à Balzac son entreprise de description et d'analyse des espèces sociales : L'Avant-Propos de la Comédie humaine vante ainsi "l'éternel honneur de Geoffroy Saint Hilaire, le vainqueur de Cuvier sur ce point de haute science» $(I, 8)$ que fut le débat entre fixisme et évolutionnisme tenu par les deux savants au printemps 1830.

Mais l'enthousiasme affiché de Balzac pour Geoffroy Saint Hilaire doit toutefois être nuancé. Ce n'est pas sans une légère forfanterie que Balzac affirme dans L'Avant-Propos qu'il était lui-même " pénétré de ce système bien avant les débats auxquels il a donné lieu » $(\mathrm{I}, 8)$ au printemps 1830 entre Cuvier et Geoffroy : le romancier suivit de bien loin la controverse ${ }^{7}$. En outre, l'intérêt de Balzac tient essentiellement à un point de la doctrine de Geoffroy : le principe d'unité de plan et de composition; pour le reste, le romancier s'en éloigne. Sur le fond, il est loin de partager les idées les plus avancées du naturaliste, en particulier sur tout ce qui touche à la question de l'évolution; et les textes plus tardifs du romancier font apparaître des principes plus proches du fixisme de Cuvier que de l'évolutionnisme de Geoffroy.

Quoiqu'essentiellement livresque, et plus discrète dans le corpus romanesque, l'influence de Cuvier semble bien avoir été décisive. Dès 1831, Balzac lui consacrait un véritable panégyrique développé dans La Peau de chagrin, qui fait du paléontologue un «Enchanteur », et même le «plus grand poète de notre siècle " $^{8}$. Plus concrètement, diverses remarques du romancier témoignent de son imprégnation des théories fixistes : ainsi dans Beatrix (1839), c'est une analogie avec le monde animal qui permet au romancier de dépeindre l'extraordinaire rigidité de la société bretonne, que les 
troubles révolutionnaires semblent n'avoir nullement modifiée: à Guérande, «le caractère d'immuabilité que la nature a donné à ses espèces zoologiques se retrouve chez les hommes ». (Béatrix, II, 640) L'exemple montre bien comment la lecture de Cuvier a offert au romancier des éléments décisifs pour penser ces " espèces sociales " qu'il s'est attaché à décrire dans le corpus romanesque.

Le positionnement théorique de Balzac dans le débat entre fixisme et évolutionnisme est cependant hésitant. Le portrait d'Esther, la courtisane juive qui inspire une folle passion à Rubempré dans Splendeurs et misères des courtisanes, suscite ainsi un curieux développement - qui coûta, d'après le propre témoignage de Balzac, « bien des peines à son auteur » : alors même que cette page doit " pose[r] peut-être de grandes questions scientifiques" (Préface d'Une fille d'Ève, II, 268), on voit l'auteur s'y perdre dans d'apparentes contradictions épistémologiques, faute de parvenir à articuler les deux modèles qui l'inspirent. . Pour justifier la fragilité psychique et surtout morale d'Esther, ainsi que sa beauté, qui condense l'essence de la beauté juive, le portrait s'appuie sur des éléments contradictoires :

Les instincts sont des faits vivants dont la cause gît dans une nécessité subie. Les variétés animales sont le résultat de l'exercice de ces instincts. Pour se convaincre de cette vérité tant cherchée, il suffit d'étendre aux troupeaux d'hommes l' observation récemment faite sur les troupeaux de moutons espagnols et anglais qui, dans les prairies de plaines où l'herbe abonde, paissent serrés les uns contre les autres, et se dispersent sur les montagnes où l'herbe est rare. Arrachez à leur pays ces deux espèces de moutons, transportez-les en Suisse ou en France : le mouton de montagne y paîtra séparé, quoique dans une prairie basse et touffue, les moutons de plaine y paîtront l'un contre l'autre, quoique sur une Alpe. Plusieurs générations réforment à peine les instincts acquis et transmis. À cent ans de distance, l'esprit de la montagne reparaît dans un agneau réfractaire, comme, après dix-huit cents ans de bannissement l'Orient brillait dans les yeux et dans la figure d'Esther. (Splendeurs et misères des courtisanes - VI, 465).

Le texte semble opérer une contradiction logique entre le principe, réaffirmé en ouverture de paragraphe, et son illustration. Alors même que la formule initiale rappelle l'idée évolutionniste selon laquelle la modification des besoins, entraînant celle des fonctions et donc des organes, a permis la diversification des espèces, l'exemple choisi, qui oppose différentes races de moutons, semble revenir à un postulat fixiste, affirmant l'immuabilité des instincts malgré la modification du milieu - en tout cas dans une temporalité brève qui serait celle du temps humain, historique, et non plus la temporalité longue du temps biologique, naturel. Alors même que le principe d'une transmission par la génération des caractères acquis ou modifiés est largement admis au XIX $x^{e}$ siècle, Balzac semble ici réaffirmer la fixité des instincts spécifiques, et leur rémanence profonde, qui autorise leur résurgence inattendue, à des siècles de distance.

Il n'est certainement pas anodin que ces considérations surviennent à l'occasion du portrait d'Esther, la courtisane juive : tous les arguments sont bons, semble-t-il, aux yeux du romancier, pour motiver son portrait physique et moral, et rattacher son héroïne à un cliché littéraire alors en vogue - quitte à verser dans une forme de déterminisme bien peu cohérente avec les théories naturalistes dont Balzac se réclame. Si les théories naturalistes lui ont offert des outils pour penser le bouleversement social dont il est le témoin, on constate que ces outils méthodologiques demeurent instrumentalisés au service de la fiction, le romancier n'hésitant pas à plier la théorie pour les besoins de son récit. Le cas d'Esther, étonnante résurgence de traits anciens, 
apparaît en effet comme un cas particulier au regard de l'humanité balzacienne, soumise à la nécessité du changement et de l'adaptation.

Cette innutrition scientifique a offert à Balzac des outils conceptuels pour penser l'évolution de la société française, ainsi que des ressources poétiques : c'est ce à quoi nous allons maintenant nous attacher. La peinture balzacienne de la société française, bouleversée par les révolutions successives, évoque avant l'heure les théories de Darwin sur le struggle for life et la nécessité de l'adaptation, que Spencer transposera du plan zoologique au plan sociologique. Balzac décrit la société comme un espace de compétition permanente, animé par une lutte violente et brutale pour la survie : « cette vie de Paris est un combat perpétuel » (Le Père Goriot - III, 121), car « une rapide fortune est le problème que se proposent de résoudre en ce moment cinquante mille jeunes gens qui se trouvent tous dans votre position. Jugez des efforts que vous avez à faire et de l'acharnement du combat. Il faut vous manger les uns les autres comme des araignées dans un pot, attendu qu'il n'y a pas cinquante mille bonnes places » (Ibid., 139). Ce tableau n'est pas sans évoquer les plus sauvages conclusions des zoologistes. Le propos se fera ailleurs plus cru : pour faire son chemin dans la société parisienne, il faut " comme sur un champ de bataille, tuer pour ne pas être tué, tromper pour ne pas être trompé, [...] déposer à la barrière sa conscience, son cœur » (III, 151), c'est-à-dire renoncer à toute forme de dignité humaine, pour se ravaler au rang de la bête fauve. La remarque vaut aussi bien pour la province : l'élimination des plus faibles y constitue un motif récurrent du roman balzacien, du Curé de Tours à Pierrette.

Le modèle zoologique offre à Balzac non seulement des outils conceptuels, mais aussi des procédés poétiques pour illustrer cette réalité dans l'œuvre. Fondées sur le principe d'analogie cher à Geoffroy saint Hilaire, les métaphores et comparaisons à référent animal permettent de suggérer dans le roman la violence intrinsèque à la vie sociale, en évoquant l'intense compétition qui se joue, dans la société comme dans le monde animal, autour de la question de la survie. Cette compétition peut se résumer, de manière un peu caricaturale, selon une opposition binaire: celle du fauve et du mouton.

L'humanité balzacienne dans sa plus haute forme s'incarne dans ces personnages de conquérants, héros ambitieux et dominateurs qui savent plier la société à leurs désirs. Presque tous les représentants de cette catégorie - Rastignac (Le Père Goriot), Rubempré (Illusions perdues, Splendeurs et misères des courtisanes), de Marsay, Vandenesse, Montrivau (La Duchesse de Langeais) - appellent la métaphore du fauve, lion ou tigre. Par-delà la terminologie à la modes, l'analogie met en lumière une capacité d'adaptation de ces protagonistes, une belle aptitude à la survie dans ce milieu hostile qu'est la société mondaine, régie par une féroce loi de compétition : en effet, le fauve connote traditionnellement la puissance, la force, tous éléments qui assurent sa domination sur le règne animal. Chez Rastignac ${ }^{10}$, la métaphore révèle une féroce ambition qui permettra au héros de s'imposer sur la société parisienne. Dans Illusions perdues, lorsque quatre dandys entrent dans la loge de Mme d'Espard pour ridiculiser Rubempré, c'est encore l'image du fauve qui permet d'opposer les conquérants, aptes à la survie sociale, au malheureux provincial, voué à être éliminé11. Mais l'image peut prendre une dimension légèrement inquiétante quand elle survient chez des héros plus ambigus, comme Vautrin dans Le Père Goriot. Doté d'un regard de fauve, - cet ancien forçat échappé du bagne a si bien prouvé sa capacité de survie que ses multiples évasions et crimes lui ont valu le surnom de « Trompe-la-Mort ». (Le Père Goriot, III, 189) 

conquérants, mais celle des vaincus, trop faibles pour s'adapter, incapables de lutter pour leur survie, c'est l'image du mouton qui surgit sous la plume de Balzac. Citons ainsi l'abbé Birotteau, «très-mouton de sa nature " (Le Curé de Tours, IV, 198), et par conséquent incapable de soupçonner l'atroce machination ourdie contre lui par l'abbé Troubert et Mlle Gamard; ou encore le vieux Schmucke, ami du cousin Pons, «pauvre cher mouton » qui sera pillé, volé et ruiné par la logeuse en qui il avait imprudemment placé sa confiance : bien avant lui, elle avait perçu la vraie nature de ses protégés, « doux comme des moutons, faciles à vivre, point défiants, de vrais enfants » (Le Cousin Pons, VII, 523). Ce combat perdu d'avance entre fauves et ovins est encore mobilisé par Balzac pour cristalliser l'échec complet de César Birotteau, et sanctionner sa folie des grandeurs attisée par les mirages de la spéculation financière: son face-à-face avec le responsable de sa ruine éveille «la sensation profonde que causerait le spectacle d'un mouton se défendant contre un tigre " (César Birotteau, VI, 218). vigoureusement la réalité d'une société bouleversée, où les lois féroces de la nature ont pris le pas sur les anciennes hiérarchies ; une société où chacun doit conquérir sa place, se trouvant ainsi inévitablement placé dans une logique de compétition permanente, luttant pour sa survie sociale. Pour les plus faibles, leur destin est tout tracé, sous forme de chanson : «Pauvres moutons, toujours on vous tondra » (César Birotteau., VII, 757). devait cependant se heurter au problème des rythmes temporels. La temporalité lente de la Nature ne coïncide pas avec le rythme de l'histoire; et cette divergence devient plus sensible après 1789 , tant les changements observés sur le plan social, politique, économique et moral révèlent l'accélération de l'Histoire. Cette donnée temporelle impose au romancier de réorienter son travail de classification: il ne peut plus se penser comme un catalogue figé, atemporel, des espèces sociales, mais doit intégrer une réflexion sur la temporalité et l'évolution.

Lvant-Propos, Balzac souligne le caractère crucial de ce facteur temporel, qui fait imploser le cadre naturaliste qu'il avait voulu donner à son entreprise en invalidant certaines de ses lois. Ce qui vaut pour les espèces animales ne vaut pas systématiquement pour les espèces sociales: «Si quelques savants n'admettent pas encore que l'Animalité se transborde dans l'Humanité par un immense courant de vie, l'épicier devient certainement pair de France, et le noble descend parfois au dernier rang social. » (I, 9). Les bornes que « la Nature a posé[es], pour les variétés animales » (I, 8) paraissent n'être plus vraiment hermétiques et imperméables lorsqu'on étudie les espèces sociales dans cette époque mouvante $d u$ premier $\mathrm{xIX}^{\mathrm{e}}$ siècle: dans ces circonstances troublées, les conditions de la vie sociale se modifient brutalement, provoquant une accélération de la sélection. Les nouveaux besoins suscités par cette société de l'argent et du profit, née de 1815, entraîne une évolution soudaine, voire la transformation des espèces sociales. Pour autant, Balzac, confronté à la réalité de la vie sociale, ne se convertit pas immédiatement à l'évolutionnisme : le constat appelle la nuance.?

Le parcours d'Eugène de Rastignac dans Le Père Goriot en témoigne : il offre un excellent exemple pour étudier l'adaptation de l'aristocratie aux nouvelles conditions de la vie sociale. Aîné d'une noble famille désargentée, Rastignac est le seul espoir de survie de sa famille, qui dépend entièrement de sa réussite parisienne : «les destinées de cinq 
personnes reposent sur [sa] tête » (III, 127) - «papa, maman, grand'tante, deux sœurs (dix-huit et dix-sept ans), deux petits frères (quinze et dix ans) » selon le calcul de Vautrin (III, 137). La conquête d'une position solide et d'une source de revenus est donc un enjeu décisif : mais celle-ci se fera sur le mode de l'adaptation, de la transformation. Pour réaliser ses ambitions, Rastignac doit en effet se convertir aux règles nouvelles qui régissent la société de la Restauration, société du paraître où la compétition se joue désormais sur des détails: "un tas de cabriolets, de bottes cirées, d'agrès indispensables, des chaînes d'or, dès le matin des gants de daim blancs qui coûtent six francs, et toujours des gants jaunes le soir » (III, 102), voilà les armes du fauve moderne.

Pour trouver sa place, Rastignac doit s'adapter: il doit reconnaître le pouvoir de l'argent, et prendre conscience du bouleversement des valeurs comme des besoins nouveaux imposés par ce monde neuf. Cette prise de conscience rejoint le constat amer de Victurnien d'Esgrignon dans Le Cabinet des Antiques : "Voilà donc, messieurs, où en sont les gentilshommes de France [...] Pour eux la grande question est d'avoir un tigre, un cheval anglais et des babioles... » (IV, 1013) Toutes ces «babioles » sont désormais devenues un enjeu crucial: en créant de nouveaux besoins, la société postrévolutionnaire que décrit Balzac engage le même mécanisme que celui observé par les naturalistes dans le monde animal, à savoir, une adaptation des fonctions. Elle impose ainsi une évolution des espèces qui opère aussi une sélection. Certains individus (les plus forts: les Rastignac, les Vautrin) s'adapteront, tandis que ceux qui refusent de renoncer aux valeurs traditionnelles de la caste sont condamnés à disparaitre. Car, c'est un fait, l'adaptation sociale de Rastignac ne peut se faire qu'au prix d'un douloureux sacrifice : celui des valeurs familiales et des usages de sa caste, qu'Eugène doit accepter de trahir pour assurer sa survie et consolider sa position sociale. Cette épreuve est évoquée à plusieurs reprises dans le roman, qui souligne les remords du héros lorsqu'il contacte sa famille ou qu'il y songe: le contraste est trop flagrant avec la société parisienne qui s'offre à ses yeux. Et surgit alors la tentation - rapidement écartée - de récuser les valeurs nouvelles : "Quand Eugène eut achevé cette lettre, il était en pleurs, il pensait au père Goriot tordant son vermeil et le vendant pour aller payer la lettre de change de sa fille.

Ta mère a tordu ses bijoux! se disait-il. Ta tante a pleuré sans doute en vendant quelques-unes de ses reliques! De quel droit maudirais-tu Anastasie? tu viens d'imiter pour l'égoïsme de ton avenir ce qu'elle a fait pour son amant! Qui, d'elle ou de toi, vaut mieux? » L'étudiant se sentit les entrailles rongées par une sensation de chaleur intolérable. Il voulait renoncer au monde, il voulait ne pas prendre cet argent. (Le Père Goriot, III, 127-28)

La nécessité de l'adaptation sera cependant plus forte que la tentation de la régression: l'éducation parisienne de Rastignac porte ses fruits, et détermine une évolution rapide :

Depuis un mois il s'était d'ailleurs développé chez Eugène autant de qualités que de défauts. Ses défauts, le monde et l'accomplissement de ses croissants désirs les lui avaient demandés. Parmi ses qualités se trouvait cette vivacité méridionale qui fait marcher droit à la difficulté pour la résoudre. (Le Père Goriot, III, 133)

Un tel itinéraire, reproduit à de nombreuses reprises dans La Comédie humaine, est-il révélateur d'une conversion de Balzac à l'évolutionnisme ? Pas totalement, me semblet-il. Certes, l'itinéraire de Rastignac, comme celui de bien d'autres ambitieux balzaciens, réalise et exemplifie ce qui parait être la loi du monde moderne : s'adapter ou disparaître. Mais cette règle affecte profondément les "espèces sociales ». Seuls quelques individus d'exception, dotés des qualités léonines (la force, la puissance), 
peuvent réaliser le sacrifice des valeurs que nous avons observé dans Le Père Goriot, et accomplir la transformation nécessaire pour s'adapter à de nouvelles conditions sociales. Toutefois, cette adaptation de quelques individus fracture le groupe plutôt qu'il n'assure sa survie : telle est bien l'amère leçon qu'il faut tirer du tableau que $L a$ Comédie humaine dresse du devenir de l'aristocratie dans la première moitié du $\mathrm{xIX}^{\mathrm{e}}$ siècle. Elle peut bien tenter de perdurer, identique à elle-même, en conservant ses valeurs et ses lois : mais cette solution, qui s'apparente à un mécanisme désespéré de survie plus qu'à une forme d'adaptation, est condamnée à l'échec par le romancier.

Les quelques obstinés qui s'attachent à maintenir les vieilles traditions aristocratiques dans un monde où ses valeurs ont été disqualifiées ne valent que comme le témoignage quasi-archéologique d'une société définitivement révolue et qui disparaîtra avec eux: que ce soit la princesse de Blamont-Chauvry, "curieuse antique », présentée comme "le plus poétique débris du règne de Louis $\mathrm{XV}$ », ou le vieux vidame de Pamiers, "autre ruine contemporaine $»^{12}$. Dans la peinture sociale qu'est La Comédie humaine, leur valeur poétique est essentielle: elle rejoint celle des "ruines " chères aux Romantiques: témoignages touchants, tragiques, de la finitude des mondes - quand leur décalage avec le monde moderne ne revêt pas une dimension comique.

L'espèce aristocratique survit-elle alors chez ces ambitieux qui, à l'instar de Rastignac, ont su s'adapter aux conditions nouvelles du monde moderne? Balzac nous invite à répondre par la négative: les valeurs aristocratiques, chez ces jeunes lions conquérants, sont à tel point mises à mal qu'elles signent, d'une autre manière, la disparition de la vieille aristocratie d'ancien régime. Ses valeurs ont été tellement transformées par les nouvelles conditions de la vie sociale sous la Restauration, qu'elle en est devenue méconnaissable.

Le devenir des espèces sociales, dans le temps bouleversé du premier XIX ${ }^{e}$ siècle, ne pouvait donc se laisser saisir dans une nomenclature fixe et synchronique: l'accélération de l'Histoire enregistrée depuis 1789 imposait à Balzac de prendre en compte, dans son entreprise naturaliste, le facteur temps, et de reconnaître les modifications qu'apportait à sa méthode la temporalité brève de l'histoire sociale et politique, qui invalide certaines lois naturelles. La prise en compte de ces données amène ainsi Balzac à confusément pressentir une question appelée à devenir cruciale par la suite: celle de la sélection naturelle, de la survie des espèces et de l'adaptation, corollaires indispensables de la réflexion sur l'évolution.

Toutefois, cet échec relatif de la théorisation scientifique que Balzac ambitionnait de réaliser dans La Comédie humaine reçoit une somptueuse compensation sur le plan romanesque - compensation dont le romancier lui-même s'est montré conscient. L'éclatement des catégories et classes sociales ouvre le règne de l'individualisme, et déploie ainsi un champ largement ouvert pour l'inspiration romanesque :

Chez nous, autrefois, le roman rencontrait aussi des éléments fort simples et peu nombreux [...] Autrefois tout était simplifié par les institutions monarchiques; les caractères étaient tranchés: un bourgeois marchand, ou artisan, un noble entièrement libre, un paysan esclave, voilà l'ancienne société de l'Europe ; elle prêtait peu aux incidents du roman. [...] Aujourd'hui l'Égalité produit en France des nuances infinies. Jadis, la caste donnait à chacun une physionomie qui dominait l'individu; aujourd'hui, l'individu ne tient sa physionomie que de lui-même. [...] Le champ social est à tous. Il n'y a plus d'originalité que dans les professions, de comique que dans les habitudes. [...] Voilà pourquoi l'auteur a choisi pour sujet de son œuvre la société française; elle seule offre esprit et spontanéité dans les 
situations normales où chacun peut retrouver sa pensée et sa nature.(Préface d'Une

Fille d'Ève, II, 263).

Une version de cet article a été précédemment publiée dans Biological Time, Historical Time. Transfers and Transformations in 19th Century Literature, Niklas Bender et Gisèle Séginger (dir.), Brill | Rodopi, 2018, « Faux-Titre », p. 241-257, https://brill.com/view/title/39098.

\section{NOTES}

1. Toutes les références à l'œuvre de Balzac sont tirées de l'édition dirigée par P. G. Castex aux éditions Gallimard, coll. La Pléiade, 1976-1981: les chiffres romains renvoient au numéro de volume, les chiffres arabes, au numéro de page.

2. Suivant l'explication donnée par Balzac dans une lettre à Mme Hanska du 26 octobre 1834 : « Les Études de mœurs représenteront tous les effets sociaux [...] La seconde assise sont les Études philosophiques, car après les effets viennent les causes. [...] Puis, après les effets et les causes, viendront les Études analytiques, dont fait partie la Physiologie du mariage, car après les effets et les causes doivent se rechercher les principes. » (Honoré de Balzac, Correspondance)

3. Texte primitif de l'Avant-propos, cité par M. Fargeaud, I, 9, $\mathrm{n}^{\circ} 2$.

4. Qui commente l'Avant-Propos de La Comédie humaine dans l'édition La Pléiade.

5. Le roman multiplie les références au Museum d'histoire naturelle - un des pensionnaires de la maison Vauquer est employé de cette institution - et l'auteur n'hésite pas à parodier le «style naturaliste » pour dresser le portrait de tel ou tel personnage. J'en veux pour exemple le portrait de Goriot, rattaché à la classe des invertébrés : «l'abus des plaisirs en faisait un colimaçon, un mollusque anthropomorphe à classer dans les Casquettifères, disait un employé au Museum, un des habitués à cachet. » (Le Père Goriot,III, 73)

6. Modeste Mignon, I, 642.

7. Madeleine Fargeaud rappelle (op. cit.) que le romancier était absent de Paris au moment des débats, séjournant à la Grenadière auprès de sa maîtresse de l'époque, Mme de Berny, ce qui l'empêcha de suivre avec attention la polémique.

8. «Vous êtes-vous jamais lancé dans l'immensité de l'espace et du temps, en lisant les œuvres géologiques de Cuvier ? Emporté par son génie, avez-vous plané sur l'abîme sans bornes du passé, comme soutenu par la main d'un enchanteur? En découvrant de tranche en tranche, de couche en couche, sous les carrières de Montmartre ou dans les schistes de l'Oural, ces animaux dont les dépouilles fossilisées appartiennent à des civilisations antédiluviennes, l'âme est effrayée d'entrevoir des milliards d'années, des millions de peuples que la faible mémoire humaine, que l'indestructible tradition divine ont oubliés et dont la cendre, poussée à la surface de notre globe, $\mathrm{y}$ forme les deux pieds de terre qui nous donnent du pain et des fleurs. Cuvier n'est-il pas le plus grand poète de notre siècle ? [...] Il est poète avec des chiffres, il est sublime en posant un zéro près d'un sept. Il réveille le néant sans prononcer des paroles grandement magiques; il fouille une parcelle de gypse, y aperçoit une empreinte, et vous crie: Voyez! Soudain les marbres s'animalisent, la mort se vivifie, le monde se déroule !» (La Peau de chagrin, X, 74-76).

9. C'est à la suite d'un « cadeau linguistique » de l'Angleterre, rappelle Balzac dans Albert Savarus, que le terme de "lion» est passé en France pour désigner les dandys et leurs recherches d'élégance : aussitôt «le lion promena dans Paris sa crinière, sa barbe et ses moustaches, ses gilets et son lorgnon tenu sans le secours des mains, par la contraction de la joue et de l'arcade sourcilière. » $(\mathrm{I}, 917)$ 
10. «C'est fatigant de désirer toujours sans jamais se satisfaire. Si vous étiez pâle et de la nature des mollusques, vous n'auriez rien à craindre ; mais nous avons le sang fiévreux des lions et un appétit à faire vingt sottises par jour. » (Le Père Goriot, III, 138)

11. «[...] monsieur de Marsay, homme fameux par les passions qu'il inspirait, remarquable surtout par une beauté de jeune fille, beauté molle, efféminée, mais corrigée par un regard fixe, calme, fauve et rigide comme celui d'un tigre : on l'aimait, et il effrayait. Lucien était aussi beau ; mais chez lui le regard était si doux, son œil bleu était si limpide, qu'il ne paraissait pas susceptible d'avoir cette force et cette puissance à laquelle s'attachent tant les femmes. » (Illusions perdues, $\mathrm{V}, 277$ )

12. La Duchesse de Langeais, V, 1010-1011.

\section{RÉSUMÉS}

Dans La Comédie humaine, Balzac entend dresser un tableau complet des " espèces sociales ", sur le modèle des nomenclatures animales établies par Buffon et Cuvier. Mais le romancier, confronté aux transformations de son siècle, se veut aussi historien. La succession des révolutions en France au XIX ${ }^{e}$ siècle le confronte à deux temporalités divergentes, celle, brève et bouleversée, de l'Homme dans l'Histoire, et celle, plus longue, de la nature. Ces révolutions, qui transforment profondément la société française, posent de façon cruciale la question de l'adaptation de l'individu dans une société en pleine mutation. Les modèles scientifiques des naturalistes, notamment le transformisme de Geoffroy Saint Hilaire, offrent au romancier un modèle théorique pour construire une nomenclature des espèces sociales en prise avec l'Histoire, et conjuguer ainsi harmonieusement modèle historique et biologique.

In La Comédie humaine, Balzac aims to provide a complete description of the various 'social species', which was modelled on the animal classifications established by Buffon and Cuvier. However, faced with the transformations that his century had undergone, Balzac the novelist also wished to be a historian. The succession of revolutions in France in the 19th century confronted him with two divergent time scales: the brief and shattered one of Man in History; and the longer one of Nature. These revolutions, which were profoundly transforming French society, raised the crucial question of the individual's adaptation to a rapidly changing society. The scientific models of naturalists, especially Geoffroy Saint Hilaire's transformism, offered the novelist a theoretical model for constructing a classification of social species caught up in history, and thus for harmoniously combining historical and biological models

\section{INDEX}

Mots-clés : évolution, espèces sociales, révolution, Balzac (Honoré de), Saint Hilaire (Geoffroy)

Keywords : evolution, sociales species, revolution, Balzac (Honoré de), Saint Hilaire (Geoffroy) 
AUTEUR

SANDRA COLLET

Laboratoire LISAA (EA 4120), Université Gustave Eiffel 